
internationales

vol. $27-n^{\circ} 1 \mid 2011$

La construction de l'altérité dans l'espace noir atlantique : Etats-Unis - France - Caraïbes - Amérique latine

\title{
Entre universalisme et multiculturalisme : politiques publiques et luttes politiques autour de l'éducation supérieure en Colombie
}

Between Universalism and Multiculturalism: Public Policies and Political

Struggles in Colombian higher Education

Entre universalismo y multiculturalismo: políticas públicas y luchas políticas en torno a la educación superior en Colombia

Oscar Quintero

\section{OpenEdition}

12 Journals

Édition électronique

URL : https://journals.openedition.org/remi/5330

DOI : $10.4000 /$ remi.5330

ISSN : $1777-5418$

Éditeur

Université de Poitiers

Édition imprimée

Date de publication : 1 juin 2011

Pagination : 71-87

ISBN : 979-10-90426-00-9

ISSN : 0765-0752

Référence électronique

Oscar Quintero, « Entre universalisme et multiculturalisme : politiques publiques et luttes politiques autour de l'éducation supérieure en Colombie », Revue européenne des migrations internationales [En ligne], vol. 27 - $n^{\circ} 1$ | 2011, mis en ligne le 01 juin 2014, consulté le 14 avril 2022. URL : http:// journals.openedition.org/remi/5330; DOI : https://doi.org/10.4000/remi.5330 


\section{Entre universalisme et multiculturalisme : politiques publiques et luttes politiques autour de l'éducation supérieure en Colombie}

\section{Oscar QUINTERO ${ }^{1}$}

\section{INTRODUCTION}

$\mathrm{Z}^{\mathrm{n}}$ Colombie, les tensions entre production et reproduction des inégalités

$\mathrm{Z}_{\text {sociales, promotion de la mobilité sociale et recompositions identitaires des }}$ étudiants ont été présentes tout au long du processus d'expansion du système d'enseignement supérieur (Parra, 1996). Depuis les années 1940, le système universitaire colombien connaît une très forte croissance : le nombre d'étudiants est passé de 2990 en 1940 à 1400000 en $2008^{2}$. Le pays dispose aujourd'hui d'un système d'enseignement supérieur très hétérogène en ce qui concerne la qualité de l'éducation, les programmes, la valeur des diplômes sur le marché du travail et dans les hiérarchies de la distinction sociale (Arango, 2006).

Dans ce contexte global, des programmes préférentiels existent, visant à l'égalité des chances dans l'accès à l'enseignement supérieur pour les minorités ethniques. Ceux-ci ciblent historiquement davantage les populations indigènes que les populations noires. Nous analyserons, d'une part, les politiques publiques dans l'enseignement supérieur adressées aux populations noires à partir des années 1990 et, d'autre part, les dynamiques sociales qui se nouent autour du multiculturalisme dans l'enseignement supérieur. Nous

1 Doctorant en Sociologie à l'Université Rennes 2, Laboratoire RESO. Membre du projet Afrodesc, ancien boursier de l'IRD. Membre du Grupo Interdisciplinario de Estudios de Género - GIEG, Centro de Estudios Sociales, Universidad Nacional de Colombia. Unidad Camilo Torres, Calle 44 No. 45-67 - Bloque B5 y B6, Ciudad Universitaria - Bogotá D.C. - Colombia ; oquinteror@ yahoo.com

2 Ministerio de Educación Nacional. http://menweb.mineducacion.gov.co/info_sector/estadisticas/ superior/dpto_total.php?consulta=mat_total. Consulté le 23 février 2010. 
aborderons ces questions par l'analyse des stratégies mises en pratique par les associations noires, les étudiants, les universités et le secteur éducatif en général.

Nous développerons l'hypothèse de travail selon laquelle, malgré l'existence d'un ensemble significatif de règles, normes et politiques dites « multiculturelles », dans la pratique se maintiennent des logiques fondées sur un modèle universaliste abstrait à partir d'une définition formelle de l'égalité entre les citoyens (première partie). Ces logiques se déclinent sous la forme de stratégies égalitaires selon des critères de classe sociale, de territoire de résidence et de performance académique. Cette tendance a suscité des revendications de plus en plus vigoureuses de la part des associations et des étudiants noirs afin de faire valoir les préceptes multiculturels dans les faits, dans l'enseignement supérieur (deuxième partie). Ainsi sont mis en lumière des processus conflictuels d'ethnicisation et de racialisation qui sont étroitement associés l'un à l'autre, jusqu'à parfois ne pas pouvoir être distingués dans le cours de la réalité sociale.

\section{LES POLITIQUES PUBLIQUES VISANT LES POPULATIONS NOIRES DANS L'ENSEIGNEMENT SUPÉRIEUR}

Une analyse approfondie des règles et normes du champ de l'enseignement supérieur nous permet d'identifier les caractéristiques des politiques et programmes à caractère multiculturel, ainsi que le processus de construction des catégories qui définissent des groupes de population en tant que groupes ethniques et leur rôle et position dans la société colombienne. Pour ce faire, nous avons privilégié une analyse des normes aux trois niveaux qui constituent le corpus principal du système législatif colombien : i) la Constitution Politique, ii) les Lois à caractère national et iii) les Décrets d'application des lois.

\section{Les populations noires dans les normes générales sur l'éducation}

D’après la Constitution Politique de 1991, «l'État reconnaît et protège la diversité ethnique et culturelle de la Nation colombienne » (Art. 7). Cette reconnaissance de la diversité culturelle cohabite avec le principe d'égalité de toutes les personnes à l'égard de la loi, en garantissant «la même protection et traitement des autorités tout en ayant les mêmes droits, libertés et opportunités sans aucune discrimination » (Art. 13). Dans le domaine de l'éducation, la Constitution établit que «les membres des groupes ethniques auront le droit à une formation qui respecte et promeut leur identité culturelle » (Art. 68). Il existe également une catégorie institutionnelle définissant les populations noires en Colombie en tant que «communautés noires » (Art. Trans. 55), laquelle sera définie par la suite dans la Loi 70 de 1993 comme "l'ensemble de familles d'ascendance afrocolombienne qui possèdent une culture propre, qui partagent une histoire et qui ont leurs propres traditions et coutumes dans la relation territoire-population lesquelles révèlent et maintiennent une conscience identitaire qui les distingue des autres groupes ethniques » (Art. 2, item 5).

D'autre part, la Loi qui réglemente le « service public de l'Enseignement Supérieur » (Loi 30 de 1992) n'établit pas de catégories ethniques qui seraient cibles de 
politiques préférentielles. Le seul critère considéré est celui de la classe sociale visant «les étudiants à faibles revenus économiques » (Art. 111). La même Loi définit les caractéristiques des étudiants susceptibles de recevoir les aides publiques : «a) excellence académique, b) manque de ressources économiques, c) distribution régionale proportionnelle au nombre d'étudiants, d) distribution adéquate pour tous les champs de la connaissance » (Art. 114).

Les critères ethniques sont donc absents de cette loi qui apparaît en décalage par rapport aux principes multiculturels de la Constitution. Ceci est compréhensible car elle est antérieure à la Loi 70 de 1993 qui constitue la plus importante norme concernant les populations noires. D'ailleurs, cette Loi 30 définit une conception « classique » du droit à l'éducation, dans le sens où la valeur mobilisée est la méritocratie, à travers les résultats scolaires et le manque de ressources économiques. Ce faisant, les critères sur lesquels elle se fonde amènent plutôt à renforcer les inégalités sociales : "l'enseignement supérieur sera accessible à ceux qui possèdent les capacités requises et accomplissent les conditions académiques exigées selon le cas » (Art. 5)3. Une telle conception se base sur l'idée d'une « égalité formelle » selon laquelle tous les candidats entreraient dans l'enseignement supérieur avec les mêmes conditions et le même capital culturel et scolaire, sans tenir compte de la réalité de la reproduction des inégalités sociales à travers l'école.

Les catégories ethniques réapparaissent sous une forme différente dans la Loi Générale d'Éducation (115 de 1994). Quoiqu'elle réglemente l'éducation primaire et secondaire, certains aspects méritent d'être abordés. Dans un premier temps, elle vise des besoins d'éducation spécifique selon les catégories de population : éducation pour les personnes handicapées ou avec des capacités exceptionnelles, éducation pour les adultes, éducation paysanne et rurale, éducation pour la réhabilitation sociale. Ainsi, l'éducation pour les groupes ethniques est définie en tant qu' " ethno-éducation » : "Cette éducation doit être liée à l'environnement, au processus productif, au processus social et culturel, avec le respect nécessaire des croyances et traditions » (Titre III, Chap. 3).

L'ethno-éducation est donc conçue pour les groupes ethniques, et « les langues vernaculaires » en deviennent une composante très importante. De plus, cette législation établit comme objectif éducatif de «promouvoir l'intérêt et le respect pour l'identité culturelle des groupes ethniques » (Art. 13), à l'adresse du reste de la société colombienne. En outre, la loi encourage la participation de représentants des « communautés noires » dans les lieux de représentation éducative au niveau départemental et national. Il faut ajouter que c'est la première fois qu'apparaît la catégorie « raizal » associée à l'idée de « communauté noire » dans un sens ethnique ${ }^{4}$. La « communauté raizal » correspondant aux personnes qui ont habité historiquement l'Archipel colombien de San Andrés situé dans la Mer des Caraïbes.

3 Souligné par nous.

4 Cette catégorie apparaît également dans la Constitution de 1991 (Art. 310) mais l'association avec les « communautés noires » n'est pas explicite. La référence aux « communautés raizales » y est liée principalement au territoire afin de différencier les « communautés natives de l'Archipel » des autres populations qui y habitent (surtout les gens de la Colombie continentale). 
Plus récemment, le « Plan Sectoriel 2006-2010» a également innové en identifiant les groupes ethniques comme des « groupes vulnérables ». Cette approche est en grande partie une adaptation des recommandations des organismes internationaux tels que la Banque Inter-Américaine de Développement et la Banque Mondiale ${ }^{5}$. Le Plan définit trois priorités politiques : i) augmenter la couverture éducative, ii) améliorer la qualité de l'éducation et iii) améliorer l'efficience du secteur éducatif. Outre le chevauchement entre les idéologies « multiculturelle » et " égalitariste », il existe une autre évolution importante dans la définition des populations noires, résidant dans le passage de la catégorie « communautés noires » à celle de « populations afrocolombiennes ». Il convient maintenant de mettre en lumière les mutations et passerelles entre les processus de racialisation et d'ethnicisation, à travers l'analyse de la normativité produite spécifiquement pour les populations noires.

\section{Analyse de la « normativité afrocolombienne »}

Pour aborder cette normativité, il paraît pertinent de prendre en compte deux éléments majeurs. Le premier est lié au contenu de la législation et à la façon dont les populations sont définies. Cela permet de comprendre comment l'État définit la « diversité », et selon quelle approche idéologique et politique elle est définie. Le deuxième élément est lié aux stratégies et mécanismes de traitement des populations noires dans l'enseignement supérieur, ce qui nous permettra d'identifier les acteurs sociaux impliqués dans les processus d'ethnicisation et racialisation.

\section{Le contenu de la législation}

La réglementation visant les populations noires est très récente en Colombie. Il existe une norme qui réglemente la création de l'Université et Centre de Recherches du Pacifique (Loi 65 de 1988), région du pays qui sera quelques années plus tard au cœur de la construction de la notion de « communautés noires ». Il faut noter que cette loi ne fait pas référence à l'ethnicité, mais propose une approche régionale. Un cas similaire est celui de la Loi 47 de 1993 établissant des normes spéciales pour le fonctionnement de l'Archipel de San Andrés sous le statut administratif de « département ». Cette loi prévoit la création d'une université départementale avec des programmes d'enseignement en rapport avec les besoins du territoire.

Comme nous l'avons évoqué, le développement législatif le plus important par rapport aux populations noires réside dans la Loi 70 de 1993. Dans le domaine de l'éducation, elle prévoit de " garantir l'accès à l'enseignement supérieur » et de "promouvoir l'ethno-éducation » dans toutes les institutions éducatives du pays. Elle inspirera et encadrera les développements postérieurs en matière d'éducation, par exemple à travers la " Commission Pédagogique des Communautés Noires » chargée de " conseiller, générer et accompagner " les initiatives éducatives des communautés noires, et de soutenir les "Chaires afrocolombiennes » à l'Université du Pacifique et dans les écoles et collèges (Décret 2249 de 1995).

5 Cf. Appel d'Offre au Projet d'Expertise Acceso, Permanencia y Graduación de la Población Diversa en la Educación Superior, Ministerio de Educación Nacional, 2007. 
Quelques années plus tard, le Décret 1627 de 1996 prévoit la création et la réglementation d'un fonds spécial pour l'éducation des communautés noires. Ce programme de crédits-bourses a pour but de garantir «l'accès et la présence durable » des étudiants des « communautés noires » dans l'éducation supérieure. Enfin, le Décret 1122 de 1998 réglemente la « Chaire d'Études Afrocolombiennes » dans tous les établissements d'éducation formelle (publics et privés) du premier et du second degré. Cette réglementation vise à " promouvoir l'intégration de la diversité culturelle nationale " à l'intérieur des institutions éducatives, à travers la promotion de la connaissance de l'histoire et les traditions culturelles des communautés noires, le respect des principes d'égalité et de non-discrimination.

\section{La catégorisation de la population par les normes}

La catégorie initiale de « communauté noire » se maintient tout au long de la réglementation, mais, dans la pratique, elle n'est plus seulement utilisée pour faire référence aux populations rurales collectives du littoral Pacifique, d'autant que d'autres catégories sont introduites telles que « communautés afrocolombiennes » ou même " groupe ethnique noir ».

La définition de la « population noire » mêlant des approches de divers ordres, reste donc ambiguë. D'un côté, l'association de la logique « raciale » - dont la couleur de la peau - à certaines caractéristiques « culturelles » fonde la définition du " groupe ethnique », ce qui est évoqué par l'usage des mots « noire(s)», «noir(s) ». De l'autre côté, une approche plus culturaliste, voire « ethnique », met en avant une identité culturelle partagée historiquement et qui est représentée par l'usage des mots « afrocolombien(s) », « afrocolombienne(s) » (à ce propos voir, Wade, 1999 et 2002 ; Hoffmann, 2002 ; Agier et Hoffmann, 1999 ; Cunin, 2003 ; Agudelo, 1999 ; Arocha, 2004). Ceci n’est pas sans incidences, également ambiguës, sur les politiques publiques et programmes institutionnels dans l'enseignement supérieur.

\section{Conception de l'Éducation}

La compréhension des processus d'ethnicisation et de racialisation à partir de l'étude des catégories institutionnelles nous amène à analyser les différentes conceptions de l'éducation qui sont en rapport avec ces catégories. De même, ces conceptions sont en rapport avec les politiques publiques établies par l'État. Ainsi, nous avons identifié deux types de conception de l'éducation liés à la construction des populations noires en tant que « groupe ethnico-racial ». La première est basée sur la reconnaissance des populations noires en tant que «minorité ethnique » et, en conséquence, sur la valorisation de ses « traits culturels » dans l'ensemble du système éducatif, ainsi que la valorisation et la récupération de son histoire dans la construction de la nation colombienne. C'est ce que nous connaissons déjà sous le terme «d'ethno-éducation ». La seconde est fondée sur le fait que les populations noires sont les victimes historiques de processus d'exclusion et de discrimination qui ont limité leurs possibilités d'accès à l'éducation et notamment à l'enseignement supérieur. Il s'agit d'une conception « égalitaire » de l'éducation ayant pour but de compenser des inégalités historiques. 


\section{Les stratégies égalitaires}

Tout d'abord, il faut noter qu'il n'existe pas une loi spécifique « d'Action Affirmative » pour les populations noires en Colombie. Pourtant, plusieurs textes officiels conçoivent et recommandent une politique de ce type, en se référant au Document Conpes 3310 de 2004 intitulé « Politique d'Action Affirmative pour la population noire ou afrocolombienne ». Le Conpes est le «Conseil National de Politique Économique et Sociale ». Quoique cette institution ne promulgue pas de décret, elle définit l'orientation de la politique nationale et de ses recommandations dépendent les plans de développement, d'investissement public et les orientations du budget général présenté au Congrès de la République. Ainsi, nous pouvons affirmer qu'il existe au moins un précédent institutionnel assez important en la matière.

Il est intéressant d'analyser la définition de la population désignée comme bénéficiaire dans ce document : " [...] les populations noires ou afrocolombiennes qui habitent le territoire national. La politique sera destinée aux habitants des niveaux 1 et 2 du Sisben ${ }^{6}$, selon les conditions de chaque programme, et à la population déplacée » (Conpes 3310, 2004 : 8). Dans une note en bas de page, le texte ajoute : "Le terme Afrocolombien reflète les niveaux de métissage à l'œuvre dans notre pays, il fait référence à l'origine et non à la couleur de la peau, et il est davantage reconnu au niveau mondial comme un concept inclusif plutôt que d'origine raciale (sic). En Colombie, les deux termes sont utilisés sur la base de l'auto-identification » (Ibid. : 8, note 7).

Cette « définition » va dans le sens de nos remarques préliminaires. Quoiqu'au niveau conceptuel, il puisse y avoir beaucoup de différences entre les catégories « Noir » et "Afrocolombien », au niveau institutionnel ces deux catégories sont considérées comme synonymes et cela ne résout pas la question de leur ambiguïté. Il est également intéressant de noter qu'une politique dite d' " action affirmative » soit centrée explicitement sur les populations les plus pauvres. Il s'agit bien ici d'une sorte de combinaison entre des critères préférentiels ethniques, mais aussi socioéconomiques. De plus, il n'est pas anodin que cette politique reprenne à grands traits les recommandations en matière de politique économique et sociale faites par les bailleurs de fonds internationaux.

La tendance est similaire concernant le «Fonds spécial de bourses pour l'enseignement supérieur des communautés noires ». Ce Fonds spécial consiste en l'attribution de crédits à un nombre de bacheliers noirs admis dans une université du pays. La dette peut être annulée intégralement à condition que l'étudiant/e soit présenté/e par une association noire reconnue par l'État et qu'il/elle accomplisse un « travail communautaire » pendant la durée de ses études.

Malgré le discours égalitaire du Fonds visant à un accès plus large des populations noires à l'enseignement supérieur, les critères précisés pour en être bénéficiaire restent assez restrictifs. La sélection des candidats se base en effet autant sur des caracté-

6 Le Sisben est le Système de Sélection de Bénéficiaires pour les Programmes Sociaux ; son but est d'identifier, à travers une enquête, la population la plus pauvre du pays. Les niveaux 1 et 2 correspondent aux personnes les plus pauvres. 
ristiques personnelles - être « afrocolombien » avec de faibles ressources économiques et de bons résultats scolaires - que sur des critères plus collectifs : être engagé/e dans le travail communautaire et être soutenu/e par une association noire. De surcroît, il faut que l'étudiant/e ait un garant propriétaire d'un bien immobilier pour avoir accès au crédit.

Les moyens du Fonds restent donc limités eu égard à son objectif de résoudre les problèmes d'inégalité d'accès à l'enseignement supérieur des populations noires. Il s'agit d'un programme très ponctuel et limité, en accord avec les politiques néolibérales en vigueur dans le pays, dont le propos est de financer plutôt la demande que l'offre des services publics. Même sa continuité a été souvent remise en cause suite au manque de financements car il dépend directement des ressources issues de la coopération internationale, ce qui a provoqué des interruptions du programme durant quelques semestres et même plusieurs années.

Divers acteurs sociaux, institutionnels et de la société civile, interviennent dans cette stratégie du Fonds spécial de bourses. D'un côté, le Ministère de l'Intérieur et son « Bureau d'Affaires des Minorités Ethniques » contrôlent le registre officiel des associations noires qui ont la faculté de soutenir les candidatures des bacheliers candidats. L'Icetex est l'institution financière chargée d'attribuer les crédits ainsi que d'administrer le Fonds. En face, se trouvent les étudiant/es et leurs familles en tant que bénéficiaires, et les associations noires qui soutiennent les étudiants et conduisent les travaux communautaires. Malgré ses limites, le Fonds est devenu un objet de revendication pour les associations noires et les étudiant/es. Ceux-ci ont développé des actions collectives de pression sur le gouvernement afin qu'il garantisse la continuité des actions du Fonds spécial avec un budget propre de l'État, en plus des ressources de la coopération internationale ${ }^{7}$. Pour l'instant il n'y a guère eu de changements dans l'envergure du programme.

Le fait de lier ce programme financier à d'autres actions de type politique a entraîné des rapports conflictuels entre les acteurs sociaux concernés. Ainsi, les organisations noires critiquent durement les institutions gouvernementales en les accusant de mettre en place des politiques multiculturelles peu ambitieuses et sans beaucoup de ressources économiques. De l'autre côté, les organismes de l'État attaquent les associations en pointant du doigt leur politisation excessive qui irait à l'encontre d'une continuité des programmes gouvernementaux. Les étudiants eux-mêmes critiquent les associations en leur reprochant leurs pratiques clientélistes pour ce qui concerne le soutien aux candidatures des bacheliers. Finalement, tous s'accordent pour critiquer les universités, notamment publiques, dont la plupart n'a pas développé de politiques spécifiques visant les étudiants noirs, même s'il s'agit d'un principe constitutionnel et réglementaire.

7 L'organisation Cimarron a fait deux manifestations en février 2008 dans les bureaux de l'Icetex et du Département National de Planification en demandant « La capitalisation et le renforcement du Fonds ». Tract de l'Association, février 2008. 


\section{LES DYNAMIQUES SOCIALES AUTOUR DE L'ENSEIGNEMENT SUPÉRIEUR : HÉRITAGES UNIVERSALISTES ET LUTTES POUR LE MULTICULTURALISME}

Les dynamiques sociales ne se limitent pas à la législation et à la politique de l'État. Il est clair que tout cet ensemble institutionnel a ouvert des possibilités d'intervention et d'expression pour d'autres acteurs sociaux. L'analyse portera maintenant sur les dynamiques sociales qui interviennent dans les processus d'ethnicisation/racialisation des populations noires dans ce contexte formellement multiculturel, mais qui présente également un fort héritage universaliste abstrait, résultat de la construction ancienne de la nation colombienne à partir du mythe républicain du métissage.

\section{Les réponses des universités : une " affirmative action » à la colom- bienne?}

Un certain nombre d'universités publiques et privées ont développé des programmes de traitement différentiel des étudiant/es noir/es. Ceci dépend non seulement de la « bonne volonté » des universités, mais répond également à une demande éducative croissante de la part des étudiant/es noir/es (Caicedo et Castillo, 2008 ; Rojas et Castillo, 2005).

À ce propos, nous allons reprendre quelques résultats d'une enquête que nous avons menée auprès des Institutions d'Enseignement Supérieur (IES) du pays. Il faut rappeler que le système colombien recouvre quatre types d'institutions : les Universités, les Institutions Universitaires, les Institutions Technologiques et les Institutions Techniques Professionnelles. L'enquête a été adressée à 307 IES (soit $93 \%$ du total) dont 127 ont répondu au questionnaire, soit un taux de réponse de $41 \%$.

Les propos de l'enquête étaient d'identifier si les IES avaient des politiques et des programmes visant des groupes de population spécifiques, et de s'interroger sur leurs caractéristiques. La première conclusion est que l'institutionnalisation des catégories ethniques, en général, et des populations noires, en particulier, est très faible dans le système d'enseignement supérieur. Il subsiste une grande invisibilité de ces populations au sein des institutions. Parmi les établissements enquêtés, la grande majorité (79\%) n'a pas de politique ou programme visant une population spécifique. Ceci signifie que seulement $21 \%$ d'entre elles ont développé au moins un programme de ce type (soit vingtsept institutions).

D'autre part, nous avons répertorié un total de 104 programmes adressés à des populations spécifiques et développés par ces vingt-sept IES, soit près de quatre programmes par institution. La moitié (cinquante-deux) des programmes étaient adressés aux « groupes ethniques » : vingt-cinq aux populations noires, vingt aux populations indigènes, cinq aux communautés « raizales », et deux aux Roms ou Gitans. Ces programmes portent sur différents aspects (l'accès, la permanence, l'achèvement des études) et rendent compte des priorités des institutions dans leurs rapports avec les populations noires. 


\section{L'accès au système}

Un premier sujet problématique, et peut-être le plus développé par les institutions, est celui de l'accès aux études supérieures. Ces programmes se basent sur la création de quotas spécifiques attribués aux étudiant/es noir/es. Selon l'institution, ces quotas peuvent être définis de deux façons. Soit en établissant un pourcentage de l'ordre de $1 \%$ ou $2 \%$ pour chaque programme d'études. Soit en définissant un nombre fixe d'étudiants qui, dans la plupart des cas, est autour d'un ou deux étudiants par programme d'études, ce nombre pouvant s'additionner ou pas au nombre total du contingent par programme ${ }^{8}$. Cela est fonction du budget, de la réglementation et des conventions signées entre chaque institution et quelques associations noires qui mènent un travail de lobbying très insistant.

La définition des quotas peut être accompagnée de davantage de flexibilité dans les conditions d'accès, soit dans l'examen d'admission exigé par certaines universités, soit dans les résultats du baccalauréat qui est un des principaux critères de sélection académique. Dans ces cas-là, la concurrence pour accéder à l'enseignement supérieur est établie entre les candidat/es noir/es, avec un contrôle des institutions pour garantir des processus plus « équitables ». Cependant une zone d'ombre demeure sur la façon dont les institutions contrôlent les variables socioéconomiques, celles-ci étant un des éléments qui peut modifier la supposée « égalité de conditions » entre les candidat/es noir/es.

Certaines universités publiques au niveau départemental ont été pionnières de ce type de programmes, surtout dans les zones du pays avec une forte proportion de population noire. Par la suite, ce sont des institutions privées qui ont adopté des programmes liés à l'accessibilité, notamment à travers des réductions des frais d'inscription oscillant entre $20 \%$ et $50 \%$ par semestre. Ceci dépend également de chaque institution et de leur budget car le financement de ces programmes est assuré, pour la plupart, par leurs seules ressources propres. Il s'agit donc, en général, d'initiatives isolées développées par certains établissements, témoignant de l'absence d'une véritable politique sectorielle.

\section{La permanence éducative}

D'autres types de programmes visent à résoudre les problèmes de désertion universitaire des étudiant/es noir/es en encourageant leur maintien dans les institutions jusqu'à la fin des études. Ces programmes se divisent selon leurs priorités en trois types d'actions :

- Des soutiens financiers et économiques. La plupart des aides et soutiens économiques concourent à des réductions dans les frais d'inscription par semestre ${ }^{9}$. Ces réductions

8 Par exemple dans une université $\mathrm{X}$ qui définit le contingent pour le programme d'agronomie de l'ordre de cinquante étudiants. Il peut arriver d'ouvrir deux quotas additionnels : soit cinquante traditionnels plus deux pour les étudiants noirs pour un total de cinquante-deux ; ou de laisser quarante-huit quotas plus deux pour les étudiants noirs afin de maintenir les mêmes effectifs de cinquante.

9 Le système d'enseignement supérieur colombien est semestriel, de sorte que la formation universitaire de « pregrado », correspondant à la Licence en France, dure dix semestres. 
peuvent varier, en général, entre $15 \%$ et $30 \%$. On rencontre des cas où ces diminutions vont jusqu'à $50 \%$ ou $60 \%$, mais cela est rare et se présente surtout lorsqu'il s'agit de proches (frères, conjoints) qui étudient dans la même institution. Les critères pour choisir les bénéficiaires des aides relèvent de la " condition ethnique » et de la " capacité de paiement » des étudiants qui est attestée par des documents officiels certifiant leur niveau socioéconomique (factures des services publics, attestation d'inscription au Sisben, déclaration de revenus, etc.). D'un point de vue ethnique, la prérogative d'attester l'« appartenance ethnique » est confiée au « Bureau d'Affaires des Minorités Ethniques » du ministère de l'Intérieur, lequel délivre des « attestations d'appartenance ethnique » aux étudiants. Un autre critère pour attribuer les aides est d'ordre académique, l'étudiant devant assurer de bons résultats aux examens avec une moyenne minimale exigée. Parfois il est aussi requis de ne jamais " rater un cours » afin de conserver le bénéfice de l'aide pour le semestre suivant. Dans d'autres cas, le critère territorial intervient également pour bénéficier d'un soutien économique. Là encore, en règle générale, ce sont les établissements eux-mêmes qui financent ces programmes, ce qui limite le montant des aides, le nombre des bénéficiaires et, par voie de conséquence, leur impact sur les besoins des étudiants. Il faut rappeler néanmoins qu'il n'existe pas d'éducation d'enseignement supérieur gratuite en Colombie. Même les universités et institutions publiques font payer des frais d'inscription en fonction des revenus familiaux. Tous les autres frais éducatifs sont assumés par les étudiants et leurs familles.

- Des soutiens scolaires. Les associations et les étudiants mettent souvent en avant la notion de « handicap scolaire » pour expliquer l'échec universitaire. C'est par ces inégalités que les acteurs sociaux expliquent le haut niveau de désertion, qui serait donc lié au retard, creusé depuis l'école, notamment dans les compétences mathématiques et linguistiques (l'espagnol mais aussi l'anglais) ${ }^{10}$. Malgré ce diagnostic et ce constat qui semblent évidents aux yeux des étudiants et des responsables associatifs, la question n'est pas prioritaire pour les institutions éducatives, et nous n'avons repéré que très peu de programmes qui abordent directement cette problématique. Les programmes de soutien scolaire brillent par leur absence et seules deux expériences existent, à l'Université du Valle (Cali), qui a d'ailleurs le plus haut quota d'accès pour étudiant/es noir/es, et à l'Université d'Antioquia (Medellín) (García, 2007).

- Vie étudiante. Nous n'avons pas trouvé beaucoup de programmes d'actions sur la vie étudiante ayant comme public spécifique les étudiant/es noir/es. Cependant, un cas mérite d'être explicité, celui d'un programme " d'intégration étudiante " dans une université privée à Cali qui consiste à «faciliter le processus d'adaptation, sensibilisation et d'intégration à l'univers universitaire des étudiants, en fonction d'une participation sociale active et efficace dans leur performance personnelle et académique $»^{11}$. Ce programme inclut des actions de formation ethno-éducative et a pour priorité " l'expression esthétique afrodescendante » à travers la production artistique, la production intellectuelle, le leadership et le travail communautaire. Ce programme présente aussi la particularité d’être

10 Entretien groupal, Université Nationale de Colombie, février 2007, Bogota.

11 Universidad Santiago de Cali, à Cali. http://virtual.usc.edu.co/bienestar/images/stories/pdf/ bienestar\%20institucional\%20una\%20mirada\%20hacia\%201a\%20acreditaci\%D3n.pdf. Consulté le 25 février 2010 . 
cofinancé par des ressources provenant de la Banque Interaméricaine de Développement et l'Office de Culture de Cali, en plus des ressources propres de l'université.

\section{L'accomplissement des études}

Un problème récurrent dans le parcours des étudiants est lié à l'achèvement des études au moment de faire leur mémoire de fin de cycle ou leur rapport de stage, selon le cas. À l'instar de la plupart des domaines précédemment cités, il existe peu d'initiatives sur ce sujet. Nous en avons trouvé un exemple dans une institution privée déjà mentionnée, à Cali, qui a signé une convention avec le Service National d'Apprentissage (SENA) afin de faciliter l'obtention de stages et plus généralement l'insertion dans l'emploi des jeunes en formation professionnelle. D'autres institutions mettent en place des stratégies liées à l'achèvement des études qui se fondent soit sur un assouplissement des conditions d'obtention du diplôme, soit sur des conventions d'emploi avec le secteur privé.

\section{La mobilisation des associations d'étudiants pour le multicultura- lisme : le cas de l'Université Nationale}

Malgré l'appareil normatif et institutionnel visant et légitimant des politiques d'action affirmative sur un critère ethnique dans l'enseignement supérieur, les dynamiques développées par les institutions du secteur restent assez rares et isolées. À cet égard, il existe un cas très intéressant qui mérite d'être analysé car il représente les contradictions et les luttes entre un multiculturalisme officiel et officieux et les héritages d'un universalisme abstrait qui a influencé la conception de la nation colombienne depuis son indépendance au début du XIXe siècle. Nous chercherons à montrer que le référentiel multiculturaliste n'a pas réussi à remettre en cause un certain nombre de principes de l'universalisme républicain. Cela est d'autant plus évident lorsqu'il s'agit de l'Université Nationale puisqu'elle incarne justement les principes du libéralisme, de la méritocratie et de l'universalité de la connaissance scientifique.

L'Université Nationale de Colombie est l'institution publique d'enseignement supérieur la plus ancienne et la plus importante du pays. Elle est définie comme " nationale, publique et de l'État $»^{12}$. Héritière des idées des Lumières, cette université a été un acteur des événements et processus fondateurs de la construction de la nation colombienne. Cette université est une des plus sélectives du pays malgré le nombre élevé d'étudiant/es qui bénéficient de son éducation : elle est passée de 23173 étudiant/es en 2001 à 40239 étudiant/es en 2009 en licence ${ }^{13}$. Ainsi, il existe une forte concurrence entre les candidat/es en raison du caractère public (les frais d'inscription sont moins élevés), du bon niveau académique et du prestige de l'Université Nationale, reconnue comme l'une des meilleures universités du pays. Chaque année cette université reçoit la candidature d'environ 100000 bacheliers pour n'en admettre que 10 000, avec des taux d'admission

12 Vicerrectoría Académica, Universidad Nacional de Colombia. La institución-Información General, Bogotá, Noviembre de 2002.

13 Carta Universitaria, Unimedios-Universidad Nacional de Colombia, No. 48, oct. 2009, p. 6. 
qui oscillent entre $10 \%$ et $12 \%$ par an ${ }^{14}$. Cette sélection est faite à travers un examen spécifique, différent de l'examen d'État que passent tous les bacheliers.

Le discours qui justifie cette forte sélection des étudiants est fondé principalement sur le mérite académique : « les meilleurs » des bacheliers sont censés être admis à l'université (Meza, 2008). À ce propos, le directeur du Bureau d'Admissions soulignait : "Dans cette institution nous ne faisons pas de distinction de race, sexe, âge ou niveau socioéconomique. Nous prenons en compte seulement les aspects académiques $\gg^{15}$.

Ce type de discours apparaît en décalage avec les politiques analysées plus haut, notamment si l'on se réfère au cadre multiculturel, d'autant qu'il s'agit de l'université « de la nation ». Nonobstant, le même directeur du Bureau d'Admissions ajoute : « ... l'université dispose du Programme d'Admission Spécial adressé aux membres des communautés indigènes, les meilleurs bacheliers des communes pauvres et les meilleurs bacheliers du pays, ceux-ci concourent sous des conditions différentes du point de vue du score d'admission ${ }^{16}$.

Comment expliquer l'existence de ce programme ouvertement différencialiste pour les populations indigènes ? Et pourquoi une telle politique n'existe-t-elle pas pour les populations noires ? Pour répondre, il faut rappeler le processus de formation de l'identité nationale après l'indépendance au XIXe siècle et jusqu'aux premières décennies du XXe siècle. Déjà à l'époque coloniale, la blancheur de la peau était fortement valorisée tandis que les caractéristiques indiennes et noires se trouvaient en bas de l'échelle des valeurs, le noir étant au-dessous de l'indien (Wade, 1997). Cette acceptation peu problématique des catégories qui étaient censées fonder " la société de castes »-même si celle-ci n'a jamais fonctionné en tant que telle - s'est maintenue lors de la construction ultérieure de la république naissante. Elisabeth Cunin (2003) a montré combien ces discours débouchaient sur une série de stéréotypes concernant les personnes noires, stéréotypes légitimés soit par les sciences exactes (racisme scientifique du XIXe siècle), soit par la force morale de la religion (la malédiction de Cham), et diffusés en parallèle des idéaux de la politique républicaine qui ne prenaient en considération que le « citoyen » idéal et non-ethnique ni racial.

Il ne faut pas oublier que les groupes indigènes ont été le modèle de l'altérité ethnique depuis l'époque de la colonie à travers l'institutionnalisation des structures administratives spécifiques comme la mita, l'encomienda et le cabildo ${ }^{17}$. Si à l'époque républicaine, la Constitution de 1886 ne fait pas mention de la diversité culturelle, quelques années plus tard, la Loi 89 de 1890 établit le statut des indigènes en tant que « mineurs

14 Revista de la Oficina Nacional de Planeación, Universidad Nacional de Colombia, No. 13. Junio de 2008, p. 49.

15 Carta Universitaria, Unimedios-Universidad Nacional de Colombia, No. 18, ago. 2006. http:// historico.cartauniversitaria.unal.edu.co/ediciones/18/historico18.html, révisé le 27 octobre 2009. Souligné par nous.

16 Carta Universitaria, No. 18, Ibid.

17 Institutions coloniales qui régissaient le système de répartition des terres (encomienda) et du travail indigène (mita) ou l'administration des villes (cabildo). 
d'âge » (Art. 40) ${ }^{18}$. Statut inférieur qui était alors arbitré par la loi, « tandis que les noirs étaient simplement ignorés » (Wade, 1997 : 46). Dit d'une façon plus simple, « les noirs ont beaucoup moins intéressé les États, les élites intellectuelles et les sociétés métisses » que les indiens (Wade, $1997: 67$ ).

Cette logique historique nous aide à comprendre l'existence à l'Université Nationale d'un programme d'admission différencié seulement pour les étudiants indigènes depuis 1986. Notons que la date du début de cette politique est antérieure au virage multiculturel de 1991. Il ne s'agit donc pas d'une politique multiculturelle, mais d'une initiative de l'État qui reflète le type d'altérité issu du colonialisme et de la construction universaliste de la nation fondée sur le mythe du métissage.

C'est justement ce discours universaliste abstrait qui a été critiqué récemment par le « Collectif d'Étudiants Universitaires Afrocolombiens » (CEUNA), qui est devenu l'organisation regroupant des étudiant/es noir/es la plus importante du pays. Cette association a acquis une dimension nationale en septembre 2007 lors de la « Première Rencontre Nationale d'Étudiants Afrocolombiens » qui a eu lieu à Bogota et qui a été très particulière par son dénouement. En effet, ce jour-là, l'assemblée des étudiants a décidé de passer à l'action en faisant une manifestation directement devant la présidence de l'Université Nationale. Après deux ou trois heures de dialogue avec la vice-présidente de l'Université, ils ont réussi à imposer l'ouverture de négociations afin de discuter d' " un système de quotas pour l'admission des étudiants afrocolombiens à l'Université Nationale ${ }^{19}$. Ces négociations ont connu beaucoup d'interruptions et ruptures, les étudiants ont été exclus du processus de définition du programme et l'université a finalement pris des décisions de façon unilatérale et sans concertation. Ainsi, le 24 juin 2009, l'Accord 013 du Conseil Supérieur Universitaire (CSU) a créé « le programme d'admission spéciale aux meilleurs bacheliers de population noire, afrocolombienne, palenquera et raizal $»^{20}$.

Une analyse détaillée du texte permet de repérer quelques éléments intéressants. De manière générale, on peut penser qu'il s'agit effectivement d'une politique différentielle basée sur des critères ethniques car sa population cible est justement une population ethnicisée institutionnellement. Dans le détail, d'autres critères jouent de façon importante : d'abord, le programme est défini structurellement à partir d'un critère territorial car le programme fonctionne via les lycées situés dans les communes pauvres avec une population majoritairement noire. L'université choisit les lycées sur la base de statistiques démographiques fournies par l'intermédiaire d'autres institutions d'État. Le deuxième critère, lié au précédent, est de type socioéconomique ou de classe sociale - car les bénéficiaires ne sont pas les Noirs en tant que tels, mais les pauvres - de même que les communes choisies sont les communes pauvres. Finalement, le critère académique intervient après les filtres ethnique, territorial et socioéconomique pour sélectionner les meilleurs étudiants.

18 Cf. http://www.icbf.gov.co/transparencia/derechobienestar/ley/1890/ley_0089_1890.html

19 Compte-rendu de la réunion par le CEUNA, le 11 octobre 2007, polycopie, 2 p.

$20 \mathrm{http} / /$ www.admisiones.unal.edu.co/es/inicio/descargas/doc_download/73-acuerdo-numero-013de-2009-afros.html 
L'accueil de l'Accord n'a été favorable ni chez les étudiants, ni chez les universitaires et intellectuels sympathisants de « la cause afro » qui ont critiqué la façon dont les étudiants ont été exclus du processus d'élaboration du programme. Les étudiants ont appris l'existence de l'Accord par hasard, alors que celui-ci était déjà signé et approuvé. Les critiques sur le fond ont été consignées dans une lettre signée par le CEUNA, par laquelle les étudiants expriment leur inconfort face aux choix faits par la direction de l'Université. Ainsi, contestent-ils le fait que l'article 3 n'ait pas repris leurs propositions : «Les candidats [inscrits par le programme spécial] passeront leur examen d'admission en concurrence avec eux-mêmes dans chaque programme d'études, [mais] l'université a approuvé une chose totalement différente : que les bénéficiaires Afros y accéderont en ayant un score supérieur ou égal au dernier admis dans TOUTE l'université, cela veut dire que les bacheliers Afros devront concourir avec l'ensemble des candidats à l'Université $\gg^{21}$.

L'enjeu ici est très important car la proposition des étudiants faisait pression sur l'université pour, d'une certaine façon, toujours admettre des étudiants noirs dans les programmes d'études, dès lors que des candidats noirs y postuleraient. Autrement dit, cela aurait obligé l'université à remplir les quotas sans tenir compte d'un critère de sélection académique comparable à celui applicable aux autres candidats relevant de la procédure commune. Au contraire, la logique choisie par l'université maintient le critère méritocratique sur lequel se base sa distinction symbolique.

L'autre point extrêmement négatif pour les étudiants est lié au quota de $2 \%$ établi par l'université : «L'Université a toujours démontré qu'elle n'a pas de volonté politique pour destiner plus de $2 \%$ additionnel à ce programme-ci. Par rapport à cela, notre position est : l'université doit approuver un quota qui soit proportionnel au total de la population Afrodescendante du pays, [c'est-à-dire] qu'il soit réservé un minimum de $10 \%$ des places à l'université dans tous les programmes d'études et dans tous les sièges ${ }^{22}$.

La quantification devient le cœur du débat lorsqu'il peut en découler des conséquences distributives et économiques pour l'université et l'État, comme c'est le cas pour l’Université Nationale. À ce propos, un universitaire reconnu dans le domaine des « études afrocolombiennes » a protesté contre le pourcentage retenu dans une tribune libre publiée par un journal de grande diffusion, en le qualifiant de « quota mesquin $»^{23}$, provoquant ainsi un débat public sur l'action affirmative, très rare jusqu'ici en Colombie. L'Accord est resté en l'état et la première promotion a débuté ses études en février 2010. Il faudra donc attendre quelque temps pour analyser ses résultats.

21 CEUNA. Comunicado a la Opinión Pública. Sobre el programa de admisión especial a mejores bachilleres de población Negra, Afrocolombiana, palenquera y raizal, 6 août 2009.

22 Ibid.

23 Jaime Arocha, La cuota mezquina, El Espectador, 10 août 2009. 


\section{CONCLUSION}

Si l'existence d'un ensemble de dispositifs légaux et réglementaires visant à l'application du principe constitutionnel de multiculturalisme en Colombie est bien réelle, le décalage subsiste entre la législation relative à l'enseignement supérieur proprement dit et la législation spécifique concernant les populations noires. Dans la normativité du secteur éducatif, l'introduction des préceptes multiculturels, liés tant à l'ethno-éducation qu'aux stratégies visant à réduire les inégalités dans l'éducation que subissent les populations noires, reste à l'ordre du jour.

Ce décalage explique que les programmes d'action affirmative pour les étudiants noirs dans la plupart des universités colombiennes restent encore peu nombreux. Les rares universités qui les ont mis en œuvre l'ont fait grâce à la " bonne volonté » des présidents et administrateurs, ou en raison des intérêts bien compris de ces établissements (surtout privés) qui veulent capter une clientèle d'étudiants noirs de plus en plus demandeurs d'éducation supérieure. Pourtant, ce qui a clairement poussé au développement du multiculturalisme dans l'enseignement supérieur a été la pression des associations et des étudiants noirs. Ceci est certain en ce qui concerne les principales universités publiques du pays. Au-delà du cas de l'Université Nationale que nous avons étudié, les universités publiques pionnières dans l'application des programmes différenciés, comme l'Université de Valle (Cali) et l'Université d'Antioquia (Medellín), ont également été le théâtre de mobilisations énergiques de la part des étudiants.

Le cas de l'Université Nationale est intéressant parce qu'elle est un symbole de l'État et, par conséquent, des politiques nationales dans l'enseignement supérieur. Comme nous l'avons souligné, dans cette université se reproduisent les imaginaires de la nation colombienne issus de l'héritage républicain ayant érigé les indigènes en modèle d'altérité, ceux-ci étant assimilés à de « bons sauvages » et des « mineurs » (en âge). Le programme différencié envers ce groupe n'obéissait pas à une valorisation de la diversité culturelle, mais plutôt aux idées républicaines et à leur paternalisme. Ainsi s'explique le grand décalage entre la date de mise en pratique du programme visant les indigènes (1986), celle de la Constitution multiculturelle (1991) et celle du programme pour les étudiants noirs en 2009 : vingt-trois ans d'écart entre les deux groupes et dix-huit ans après la reconnaissance solennelle du caractère multiculturel de la nation.

Cette situation est archétypale des luttes pour la mise en place d'un multiculturalisme qui ne soit pas qu'un principe constitutionnel restant lettre morte, mais bien une réalité concrétisée par des politiques publiques et ayant des conséquences sur la façon dont se construit la société colombienne. Cependant, une telle orientation rencontre bien des résistances de la part des institutions universitaires et, par extension, de l'ensemble du champ éducatif. Peut-être parce que celui-ci se fonde historiquement sur des valeurs universalistes abstraites de mérite et de mobilité sociale, et parce que le fait de donner des « avantages » à certains peut délégitimer justement ces idéaux. 


\section{Références bibliographiques}

AGIER Michel et HOFFMANN Odile (1999) Les terres des communautés noires dans le Pacifique colombien. Interprétations de la loi et stratégies d'acteurs, Problèmes d'Amérique Latine, 32, pp. 17-42.

AGUDELO Carlos (1999) Colombie : changement constitutionnel et organisation des mouvements noirs, Problèmes d'Amérique Latine, 32, pp. 43-51.

ARANGO Luz Gabriela (2006) Jóvenes en la Universidad: Género, clase e identidad profesional, Bogotá, Siglo del Hombre Editores, Universidad Nacional de Colombia, 442 p.

AROCHA Jaime (2004) Ley 70 de 1993: utopía para afrodescendientes excluidos, in Jaime Arocha Comp., Utopía para los excluidos. El multiculturalismo en África y América Latina, Bogotá, Universidad Nacional de Colombia, Facultad de Ciencias Humanas, Centro de Estudios Sociales - CES - , pp. 159-178.

CAICEDO José Antonio et CASTILLO Elizabeth (2008) Indígenas y Afrodescendientes en la universidad colombiana: Nuevos sujetos, viejas estructuras, Cuadernos Interculturales, 6 (10), pp. 62-90.

CONPES 3310 (2004) Politica de Acción Afirmativa para la Población Negra o Afrocolombiana, Bogotá, Consejo Nacional de Política Económica y Social, Departamento Nacional de Planeación, Ministerio del Interior y de Justicia, República de Colombia, 43 p.

CUNIN Elisabeth (2003) Identidades a flor de piel. Lo «negro» entre apariencias y pertenencias: categorías raciales y mestizaje en Cartagena, Bogotá, ICANH, Universidad de los Andes, IFEA, Observatorio del Caribe Colombiano, 367 p.

GARCÍA Andrés (2007) Políticas étnicas afrocolombianas en educación superior: dinámicas identitarias en la Universidad de Antioquia, in Claudia Mosquera y Luis Barcelos Éds., Afroreparaciones: Memorias de la esclavitud y justicia reparativa para negros, afrocolombianos y raizales, Bogotá, Universidad Nacional de Colombia, CES, pp. 661-690.

HOFFMANN Odile (2002) Conflictos territoriales y territorialidad negra. El caso de las comunidades afrocolombianas, in Claudia Mosquera, Mauricio Pardo et Odile Hoffmann Éds., Afrodescendientes en las Américas. Trayectorias sociales e identitarias, 150 años de la abolición de la esclavitud en Colombia, Bogotá, Universidad Nacional de Colombia, Instituto Colombiano de Antropología e Historia, Institut de Recherche pour le Développement, Instituto Latinoamericano de Servicios Legales Alternativos, pp. 351-368.

MEZA Luis Guillermo (2008) Reproducción de las desigualdades sociales y raciales en la UN: los Afrocolombianos, Trabajo de Grado de Sociología, Bogotá, Universidad Nacional de Colombia, $74 \mathrm{p}$.

PARRA Rodrigo (1996) Escuela y modernidad en Colombia. Tomo IV, La Universidad, Bogotá, Fundación FES, Fundación Restrepo Barco, Tercer Mundo Editores, 265 p.

ROJAS Axel et CASTILLO Elizabeth (2005) Educar a los otros. Estado, politicas educativas y diferencia cultural en Colombia, Popayán, Editorial Universidad del Cauca, 158 p.

WADE Peter (2002) Introduction: The Colombian Pacific in Perspective, The Journal of Latin American Anthropology, 7 (2), pp. 2-33.

WADE Peter (1999) La population noire en Amérique Latine : multiculturalisme, législation et situation territoriale, Problèmes d'Amérique Latine, 32, pp. 3-16.

WADE Peter (1997) Gente negra, nación mestiza. Dinámicas de las identidades raciales en Colombia, Bogotá, Siglo del Hombre Editores, Ediciones Uniandes, 488 p. 


\title{
Entre universalisme et multiculturalisme : politiques publiques et luttes politiques autour de l'éducation supérieure en Colombie
}

\author{
Oscar Quintero
}

Cet article propose d'analyser les politiques publiques destinées à promouvoir la présence des populations noires dans le système d'enseignement supérieur colombien à partir des années 1990, ainsi que les réponses à ces politiques de la part des associations noires. Nous développerons l'hypothèse selon laquelle, malgré l'existence de réglementations et de programmes dits « multiculturels ", les logiques fondées sur le modèle de l'égalité entre les citoyens sont toujours d'actualité et se concrétisent par des propositions d'égalité des chances définies notamment à partir des critères de classe sociale ou de territoire. Pour autant, cette tendance s'accompagne d'une mobilisation croissante de la part des associations noires et des étudiants concernés afin de faire respecter et appliquer les principes multiculturels dans l'université. Ils élaborent des revendications et des propositions qui dépassent le répertoire d'action imposé par la réglementation existante et proposent un profond changement de la société colombienne.

\section{Between Universalism and Multiculturalism: Public Policies and Political Struggles in Colombian higher Education}

\section{Oscar Quintero}

This article aims at analyzing the public policies in higher education that focus on Black people, and the reaction of Black organizations to such policies since the 1990s in Colombia. The central idea is that despite existing "multiculturalist" legal frameworks and programs, there are still a number of practices based on the idea of equality among citizens, specifically grounded on the criteria of social background or territory. This approach has generated a growing demand from Black student organizations to implement multicultural principles in universities through proposals that seek to transcend the spectrum of action imposed by existing regulations and that promote a more radical change in Colombian society.

\section{Entre universalismo y multiculturalismo: políticas públicas y luchas políticas en torno a la educación superior en Colombia}

\section{Oscar Quintero}

Este artículo propone analizar las políticas públicas en educación superior dirigidas hacia las poblaciones negras en Colombia desde la década de los 90 y las respuestas a dichas políticas por parte de las organizaciones negras. Desarrollaremos la hipótesis de trabajo según la cual a pesar de existir una significativa reglamentación y programas bajo la etiqueta de «multiculturales», en la práctica siguen rigiendo lógicas basadas en el modelo de la igualdad entre los ciudadanos, las cuales son concretizadas mediante propuestas de búsqueda de acceso a igualdad de oportunidades que son definidas bajo criterios de clase social o territorio, principalmente. Esta perspectiva ha generado una creciente demanda por parte de organizaciones negras y estudiantiles para hacer realidad las devisas multiculturales en la universidad mediante propuestas que buscan trascender el espectro de acción impuesto por la reglamentación existente y que propenden por un cambio más profundo de la sociedad colombiana. 